\title{
Guest Editorial \\ Spectrum Sharing and Aggregation for Future Wireless Networks, Part II
}

\author{
Theodoros A. Tsiftsis, Senior Member, IEEE, Guoru Ding, Senior Member, IEEE, \\ Yulong Zou, Senior Member, IEEE, George K. Karagiannidis, Fellow, IEEE, \\ Zhu Han, Fellow, IEEE, and Lajos Hanzo, Fellow, IEEE
}

$\mathbf{W}$ ELCOME to the second one in the sequel of three IEEE JSAC special issues on Spectrum Sharing and Aggregation for Future Wireless Networks. In recognition of the fact that a substantial amount of submissions have been received in response to the call for papers, the decision has been made to publish three issues on the cutting-edge advances in spectrum sharing and aggregation. The first issue was published in October 2016 with 20 papers. This is the second issue with 19 papers, covering a feast of diverse topics asampled below.

The first paper, entitled "Sequence based channel hopping for dynamic spectrum sharing in cognitive radio network," designs three channel hopping protocols both for synchronous and asynchronous scenarios of cognitive radio networks for guaranteed 'rendezvous' of pairs of users in all available channels. Extensive comparisons are performed to show that the proposed protocols outperform the existing ones both in terms of their throughput, their maximum time to rendezvous as well as in terms of the average time to rendezvous.

The second paper, namely "Understanding the current operation and future roles of wireless networks: Co-existence, competition and co-operation in the unlicensed spectrum bands," presents an overview of how the co-existence and co-operation of Wi-Fi and classic cellular networks in the unlicensed spectral band improves the overall capacity of heterogeneous wireless networks. It also reveals the challenges

T. A. Tsiftsis is with the School of Engineering, Nazarbayev University, Astana 010000, Kazakhstan, and also with the Technological Educational Institute of Central Greece, 35100 Lamia, Greece (e-mail: theodoros.tsiftsis@nu.edu.kz; tsiftsis@teiste.gr).

G. Ding is with the College of Communications Engineering, PLA University of Science and Technology, Nanjing 210007, China, and also with the National Mobile Communications Research Laboratory, Southeast University, Nanjing 210018, China (e-mail: dr.guoru.ding@ieee.org).

Y. Zou is with the School of Telecommunications and Information Engineering, Nanjing University of Posts and Telecommunications, Nanjing 210003, China (e-mail: yulong.zou@njupt.edu.cn).

G. K. Karagiannidis is with the Electrical and Computer Engineering Department, Aristotle University of Thessaloniki, 54124 Thessaloniki, Greece (e-mail: geokarag@auth.gr).

Z. Han is with the Department of Electrical and Computer Engineering, University of Houston, Houston, TX 77005 USA (e-mail: zhan2@uh.edu).

L. Hanzo is with the School of Electronics and Computer Science, University of Southampton, Southampton, SO17 1BJ, U.K. (e-mail: 1h@ecs.soton.ac.uk).

Digital Object Identifier 10.1109/JSAC.2016.2616287 posed by new applications, such as machine-to-machine communications and the Internet of Things. Furthermore, it brings together two the major regulatory approaches, such as those by the UK's Ofcom and that of the European Commission, which currently represent influential efforts in terms of providing spectrum assignment authorities with robust spectrum sharing frameworks. The authors also discuss a number of compelling policy definition tools likely to be implemented.

The third paper advocates "Digitally controlled analog wideband interference cancellation for in-device spectrum sharing and aggregation" and presents a feasibility analysis of analog wideband self-interference cancellation relying on their digitally controlled analog adaptive filter. The authors analyze the key issues and concerns in analog interference cancellation and propose novel solutions for cancelling the self-interference with the aid of a low-complexity single-tap cancellation circuit. The results suggest that the proposed selfinterference cancellation approach facilitates the simultaneous operation of both licensed and unlicensed technologies in the adjacent spectrum within a single device. This facilitates graceful spectrum sharing and bandwidth aggregation between licensed and unlicensed technologies.

The fourth paper, entitled "Flexible dual-connectivity spectrum aggregation for decoupled uplink and downlink access in $5 G$ heterogeneous systems," proposes and investigates the concept of flexible cell association in dual-connectivity scenarios, where users are able to aggregate resources from more than one serving cell. In this setup, the preferred association policies for the uplink may differ from those for the downlink, thereby allowing for a truly decoupled access. The beneficial dual-connectivity association regions of decoupled access are derived with the aid of stochastic geometry and the resultant performance is evaluated in terms of the attainable capacity gains over the conventional access policies relying on the downlink received power.

The fifth paper, namely "Secondary user access control (SUAC) in cognitive radio networks" investigates the issues of secondary user access control in the context of protecting spectrum sharing/aggregation against unauthorized secondary users (UA-SUs). Rather unconventionally, a jamming signal is injected in order to degrade the spectrum sensing performance of the unauthorized secondary users, 
while reliable spectrum sensing is ensured for the authorized secondary users with the aid of a jamming cancellation method.

The sixth paper, which is entitled as "QoS-aware admission control and resource allocation in underlay device-to-device spectrum-sharing networks", investigates the spectrum sharing problem in device-to-device networks by putting forward an optimization framework that considers multiple coupled factors, including mode selection, admission control, partner assignment, and power allocation. The authors focus their attention on designing cost-efficient and easy-to-implement algorithms.

The seventh paper is focused on "A Q-learning framework for user QoE enhanced self organizing spectrally effcient network using a novel inter-operator proximal spectrum sharing" and proposes a novel spectrum sharing paradigm referred to as Inter-Operator Proximal Spectrum Sharing (IOPSS), where a base station (BS) intelligently offloads users to the neighboring BSs for the sake of enhancing the users' QoE and spectral resource utilization. The authors also propose a generic Q-Learning Framework for a BS to dynamically determine its load-dependent spectral needs and to efficiently share its spectrum. The proposed solutions result in a spectral-efficient self-organizing network of BSs.

The eighth paper, entitled "On the value of beamforming and coordination for spectrum sharing in mmWave cellular networks," investigates to what extent spectrum sharing in mmWave networks supported by multiple cellular operators is a viable alternative to traditional dedicated spectrum allocation. Specifically, the authors develop a general mathematical framework for characterizing the performance gain that the users can experience by spectrum sharing as a function of the specific beamforming technique used, operator coordination, bandwidth, and infrastructure sharing scenarios.

The ninth paper advocates "Near-optimal routing protection for in-band software-defined heterogeneous networks" and investigates optimal routing protection invoked for the controlplane traffic in software defined heterogeneous networks. This paper formulates a weighted cost-minimization problem, where the traffic load balancing and control-channel setup cost are jointly considered and proposes a near-optimal Markov approximation based approach to deal with the problem. The authors also extend their solution to an online scenario that handles a single-link failure. Both theoretical analysis and experimental simulation are performed to show that the proposed algorithm exhibits a rapid convergence and a high resource utilization efficiency.

The tenth paper, namely "Gains of restricted secondary licensing in millimeter wave cellular systems", models a mmWave cellular system where an operator that owns an exclusive primary license for a certain band can sell a restricted secondary license of the same band to another operator. The secondary network has to obey a restriction in terms of the maximum interference imposed by it on the original network. Using stochastic geometry, the authors derive expressions both for the coverage and for the rate of both networks, and establish the feasibility of secondary licensing in licensed mmWave bands. To highlight the economic trade-offs, this paper considers a revenue-pricing model for both operators in the presence of a central licensing authority. The results show that the original operator and central network authority can indeed benefit from secondary licensing, provided that the maximum interference threshold is beneficially adjusted.

The eleventh paper reports on advances in "Convolutive superposition for multicarrier cognitive radio systems" and presents a spectrum-sharing scheme conceived for multicarrier cognitive radio networks, where the secondary user convolves its block-precoded symbols with the received primary user signal in the time-domain, which justifies the terminology of convolutive superposition. An informationtheoretic analysis of the proposed scheme is developed, which considers different amounts of channel state information at the secondary transmitter, as well as different precoding strategies conceived for the secondary user.

The twelfth paper is entitled "Design and performance analysis of a fairness-based license-assisted access and resource scheduling scheme", which proposes a fairnessbased licensed-assisted access and resource scheduling scheme for guaranteeing a channel access teechniques capable of maintaining fairness between long term evolution-advanced and WiFi systems in the unlicensed band. The optimal contention window size of the proposed scheme is determined for various density ratios between the LTE and WiFi users. A novel resource scheduling approach based linear programming technique is put forward by maximizing the utility function for the sake of improving the both the user experience and the spectral efficiency. The theoretical proofs and simulation results verify the efficiency of the proposed scheme in terms of the achievable throughput, spectral efficiency, delay and packet loss ratio.

The thirteenth paper offers a treatise on the subject of "A space-time analysis of LTE and Wi-Fi inter-working" and proposes a new framework for analyzing the network performance of several inter-working strategies in the context of the LTE and the Wi-Fi standards. The proposed framework considers both the LTE system and the family of Wi-Fi systems in the context of both downlink and uplink transmissions. Both the time- and the spatial-domain interference effects are considered. Moroever, the authors analyze the performance of an architecture, where Wi-Fi coexists with an ideal duplex carrier-sence multiple access system, with the uplink being supported by the LTE and downlink by the Wi-Fi system.

The fourteenth paper, "Users first: Service-oriented spectrum auction with a two-tier framework support", develops a service-oriented spectrum auction scheme relying on a two-tier architecture in order to support the end-users with the benefits of the spectrum market without joining the auction themselves in order to conserve their limited battery power and to take into account their limited computational and communications capability. In Tier I, this paper introduces several secondary service providers in order to support the endusers with the aid of compelling services by using purchased licensed bands. In Tier II, this paper proposes a pair of social welfare maximization auction mechanisms. 
The fifteenth paper has the eloquent title of " $M^{3}$-STEP: Matching based multi-radio multi-channel spectrum trading with evolving preferences". MOre explicitly, it introduces a matching based multi-radio multi-channel spectrum trading $\left(\overline{\mathrm{M}}^{3}\right.$-STEP) scheme, which relies on a so-called conflict graph for characterizing the interference relationships amongst the secondary users and formulates the centralized primary users' revenue maximization problem under multiple constrains. This paper also develops algorithms for solvking the problem via dynamic matching in the face of evolving preferences and proves its pairwise stability.

The sixteenth paper is entitled as "Secure transmission in cognitive satellite terrestrial networks", which investigates the physical layer security of a satellite network, whose downlink spectral resource is shared with a terrestrial cellular network. This paper formulates a constrained optimization problem for maximizing the instantaneous rate of the terrestrial user, while satisfying the interference probability constraint of the satellite user. Then, the authors develop a pair of beamforming schemes, namely hybrid zero-forcing and partial zero-forcing in order to solve the optimization problem for realisitic scenarios associated with imperfect and statistical channel state information, respectively. Moreover, this paper also studies the secrecy performance of the legitimate satellite user by considering both passive and active eavesdropping scenarios.

The seventeenth paper, "Spectrum sensing and throughput analysis for cognitive two-way relay networks with multiple transmit powers", investigates a cognitive two-way relay network, where the primary users transmit data at multiple power levels. For this network, the authors design a cooperative softcombination-based spectrum sensing scheme and perform the throughput analysis of both the primary user and the secondary user.

The penultimate paper entitled "Fundamental limits of spectrum sharing full-duplex multicell networks" studies the degrees of freedom of full-duplex multicell networks that share the spectrum among multiple cells. The authors propose novel interference management strategies for establishing the sum degrees of freedom, taking into account various sources of interferences caused by both spectrum sharing and full-duplex communications. Numerical evaluations are used for demonstrating that spectrum sharing and full-duplex communications are capable of significantly improving the attainable throughput over that of conventional cellular networks, especially for a network supporting a large number of users and/or cells.

The closing paper, namely "Inter-technology coexistence in a spectrum commons: A case study of Wi-Fi and LTE in the $5 \mathrm{GHz}$ unlicensed band", presents a systematic performance evaluation of different spectrum sharing schemes designed for inter-technology coexistence in a spectrum commons. The main contributions of this paper are threefold. Firstly, the authors propose a general framework for the transparent comparative analysis of spectrum sharing mechanisms in time and frequency, by studying the effect of the key system parameters. Secondly, the authors propose a novel throughput and interference model for inter-technology coexistence, integrating per-device specifics of different distributed MAC sharing mechanisms in a unified network-level perspective. Thirdly, the authors present a case study of IEEE 802.11n Wi-Fi and LTE in the $5 \mathrm{GHz}$ unlicensed band, in order to obtain generalizable insight into their coexistence in a spectrum commons.

Recent Trends in the Evolution of Spectrum Sharing: Based on the papers in this issue some clustering of the contributions may be observed, with a focal point surrounding the following research aspects:

1) the specific spectrum sharing techniques investigated, including cognitive radios, multi-operator solutions, device-to-device sharing, auctions, etc;

2) the particular system contexts considered, as exemplified by LTE, WiFi, mm-Wave, satellite and diverse software defined networking scenarios, etc;

3) the novel optimization tools invoked for improving the techniques mentioned under 1), such as Q-learning;

4) the fundamental limits.

To elaborate a little further on the above-mentioned four aspects:

1) The specific spectrum sharing techniques, such as dual connectivity as well as decoupling the uplink and downlink, indeed exhibit substantial service-quality benefits, since the base station of a large terrestrial-cell typically has a higher downlink transmit power than that of a small base station, whilst the low-power handsets may transmit in the uplink to the small-cell base station. Cognitive radio design issues continue to attract substantial research attention also in this issue, including their secondary user support as well as their device-to-device communications aspects. The consideration of sectrum sensing and two-way relaying as well as the $\mathrm{mm}$-Wave scenarios have also attracted special attention.

2) The system contexts investigated included LTE, WiFi, $\mathrm{mm}$-Wave and satellite as well as software defined networking scenarios, all of which tend to have different propagation scenarios and bitrates, whilst supporting diverse services. Indeed, this is one of the reasons why their spectrum sharing tends to be beneficial.

3) Somewhat unexpectedly, the choice of optimization tools invoked for improving the techniques mentioned under 1) remained limited in this issue, even though the demanding optimization problems considered call for powerful multi-component optimization procedures. We might speculate that this was perhaps the consequence of having a recent JSAC special issue on the application of game-theoretic optimization approaches.

4) Similarly, further communication theory research is needed on the subject of fundamental limits. Hence this paucity of powerful multi-component optimization tools and the lack of network-theoretic analysis may indeed require further research by You - valued colleague.

Once again, we hope that through the selection of the articles in our sequel of three special issues, namely the October 2016 issue, the current issue and the forthcoming third issue, we can stimulate further in-depth discussions and new contributions to the related areas. 


\section{ACKNOWLEDGMENT}

We would like to thank all the authors who submitted their valuable contributions to this special issue. They provided both the reviewers and editors with a fascinating snapshot of the range of ongoing research in the area of spectrum sharing and aggregation. We are equally grateful to all the reviewers, who were very responsive to our repeated reminders about staying on schedule. Their critical comments and suggestions to the authors contributed substantially to the quality of the final 'product'. We are also indebted to Prof. Muriel Medard, JSAC Editor-in-Chief, to the Executive Editor Laurel Greenidge and to the Senior Editor Prof. Max Costa for the encouragement they have provided.

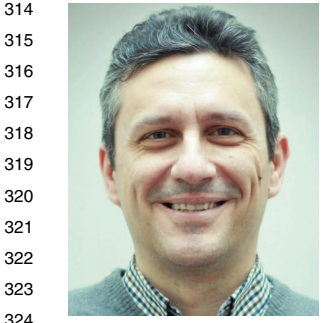

Theodoros A. Tsiftsis (S'02-M'04-SM'10) was born in Lamia, Greece, in 1970. He received the B.Sc. degree in physics from the Aristotle University of Thessaloniki, Greece, in 1993, the M.Sc. degree in digital systems engineering from Heriot-Watt University, Edinburgh, U.K., in 1995, the M.Sc. degree in decision sciences from the Athens University of Economics and Business, Greece, in 2000, and the $\mathrm{Ph} . \mathrm{D}$. degree in electrical engineering from the University of Patras, Greece, in 2006. He joined the Department of Electrical Engineering, Technological Educational Institute of Central Greece, in 2010. He is currently an Associate Professor of communication technologies with the Department of Electrical and Electronic Engineering, School of Engineering, Nazarbayev University, Astana, Kazakhstan. He has authored or co-authored over 100 technical papers in scientific journals and international conferences. His research interests include the broad areas of cooperative communications, communication theory, wireless communications, and optical wireless communication systems.

Dr. Tsiftsis was a member of the Editorial Boards of the IEEE TRANSACTIONS ON VEHICULAR TECHNOLOGY and the IEEE COMMUNICATIONS LETTERS. He acts as a reviewer of several international journals. He is currently an Area Editor of the Wireless Communications II of the IEEE TRANSACTIONS ON COMMUNICATIONS.

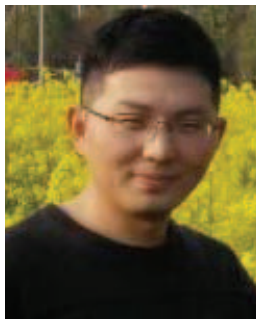

Guoru Ding (S'10-M'14-SM'16) received the B.S. degree (Hons.) in electrical engineering from Xidian University, Xi' an, China, in 2008, and the $\mathrm{Ph} . \mathrm{D}$. degree (Hons.) in communications and information systems from the College of Communications Engineering, Nanjing, China, in 2014. Since 2014, he has been an Assistant Professor with the College of Communications Engineering, and also a Research Fellow with the National High Frequency Communications Research Center of China. Since 2015, he has been a Post-Doctoral Research Associate with the National Mobile Communications Research Laboratory, Southeast University, Nanjing. His research interests include cognitive radio networks, massive MIMO, machine learning, and big data analytics over wireless networks.

Dr. Ding was a technical program committee member of a number of international conferences, including the IEEE Global Communications Conference, the IEEE International Conference on Communications, and the IEEE Vehicular Technology Conference (VTC). He currently serves as a Guest Editor of the IEEE JOURNAL ON SELECTED AREAS IN COMMUNICATIONS and an Associate Editor of the KSII Transactions on Internet and Information Systems. He is a Voting Member of the IEEE 1900.6 Standard Association Working Group. He was a recipient of the Best Paper Awards from the EAI MLICOM 2016, the IEEE VTC 2014-Fall, and the IEEE WCSP 2009.

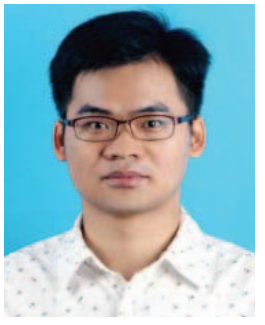

Yulong Zou (SM'13) received the B.Eng. degree in information engineering from the Nanjing University of Posts and Telecommunications (NUPT), Nanjing, China, in 2006, the first Ph.D. degree in electrica engineering from the Stevens Institute of Technology, NJ, USA, in 2012, and the second Ph.D. degree in signal and information processing from NUPT, in 2012. He is currently a Full Professor and the Doctoral Supervisor with NUPT.

His research interests span a wide range of topics in wireless communications and signal processing, including the cooperative communications, cognitive radio, wireless security, and energy-efficient communications. He is currently serving or has served as an Editor of the IEEE COMMUNICATIONS SURVEYs \& TUTORIALS, the IEEE COMmunications LetTers, IET Communications, and the China Communications. He was a recipient the 9th IEEE Communications Society Asia-Pacific Best Young Researcher in 2014. He was a co-recipient of the Best Paper Award at the 80th IEEE Vehicular Technology Conference in 2014.

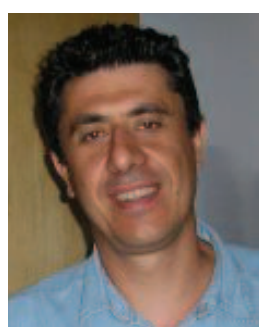

George K. Karagiannidis (M'96-SM'03-F'14) was born in Pythagoreio, Greece. He received the University Diploma and the Ph.D. degrees in electrical and computer engineering from the University of Patras in 1987 and 1999, respectively. From 2000 to 2004, he was a Senior Researcher with the Institute for Space Applications and Remote Sensing, National Observatory of Athens, Greece. In 2004, he joined the faculty of the Aristotle University of Thessaloniki, Greece, where he is currently a Professor with the Electrical and Computer Engineering Department and the Director of the Digital Telecommunications Systems and Networks Laboratory. He is also an Honorary Professor with South West Jiaotong University, Chengdu, China.

His research interests are in the broad area of digital communications systems with emphasis on wireless communications, optical wireless communications, wireless power transfer and applications, molecular communications, communications and robotics, and wireless security.

He has authored or co-authored over 400 technical papers published in scientific journals and presented at international conferences. He is also the author of the Greek edition of a book on telecommunications systems and the co-author of the book Advanced Optical Wireless Communications Systems (Cambridge Publications, 2012). He has been involved as the general chair, the technical program chair, and a member of technical program committees of several IEEE and non-IEEE conferences. He was an Editor of the IEEE TRANS ACTIONS ON COMMUNICATIONS and the EURASIP Journal of Wireless Communications and Networks, a Senior Editor of the IEEE COMMUNiCATIONS LeTtERs, and a Guest Editor of the IEEE JOURnAL Selected Areas in Communications. From 2012 to 2015, he was the Editor-in Chief of the IEEE COMMUNICATIONS LETTERS.

Dr. Karagiannidis has been selected as a 2015 Thomson Reuters Highly Cited Researcher and he was listed in the Thomson Reuters 2015 World's Most Influential Scientific Minds. 


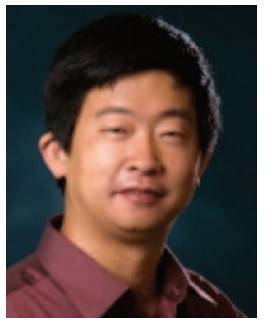

Zhu Han (S'01-M'04-SM'09-F'14) received the B.S. degree in electronic engineering from Tsinghua University in 1997, and the M.S. and Ph.D. degrees in electrical engineering from the University of Maryland, College Park, MD, USA, in 1999 and 2003, respectively. From 2000 to 2002, he was a Research and Development Engineer with JDSU, Germantown, MD, USA. From 2003 to 2006, he was a Research Associate with the University of Maryland. From 2006 to 2008, he was an Assistant Professor with Boise State University, Boise, ID, USA. He is currently an Associate Professor with the Electrical and Computer Engineering Department, University of Houston, Houston, TX, USA. His research interests include wireless resource allocation and management, wireless communications and networking, game theory, wireless multimedia, security, and smart grid communication.

Dr. Han has been an IEEE Distinguished Lecturer since 2015. He was the MAC Symposium Vice Chair of the IEEE Wireless Communications and Networking Conference in 2008. He was the Guest Editor of the Special Issue on Cooperative Networking Challenges and Applications (IEEE JOURNAL on Selected Areas in Communications) Fairness of Radio Resource Management Techniques in Wireless Networks (EURASIP Journal on Wireless Communications and Networking), and the Special Issue on Game Theory (EURASIP Journal on Advances in Signal Processing). He has been an Associate Editor of the IEEE TRANSACTIONS ON WIRELESS COMMUNICATIONS since 2010. He co-authored eight papers that received the IEEE Best Conference Paper Awards. He was a recipient of the NSF CAREER Award in 2010. He was also a recipient of the 2011 IEEE Communications Society Fred W. Ellersick Prize, the 2015 EURASIP Best Paper Award for the Journal on Advances in Signal Processing, and the 2016 IEEE Leonard G. Abraham Prize in communications systems (Best Paper Award for the IEEE JOURNAL ON SELECTED AREAS IN COMMUNICATIONS).

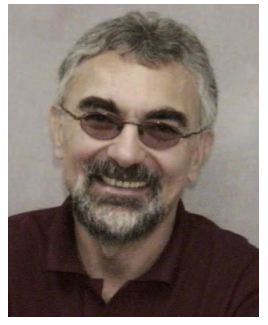

Lajos Hanzo (M'91-SM'92-F'04) received the master's degree in electronics in 1976, the $\mathrm{Ph} . \mathrm{D}$. degree from the Technical University of Budapest in 1983, and the D.Sc. degree in 2004. From 2009 to 2012, he was a Chaired Professor with Tsinghua University, Beijing, China. Since 1986, he has been with the University of Southampton, U.K. During his 40-year career in telecommunications, he has held various research and academic posts in Hungary, Germany, and the U.K. Since 1986, he has been a member of academic staff with the School of Electronics and Computer Science, University of Southampton, U.K., where he currently holds the Chair in telecommunications and is also the Head of Southampton Wireless. He has co-authored 20 John Wiley/IEEE Press books, over 10000 pages on mobile radio communications, and authored 1600 research contributions in the IEEE Xplore. He has also organized and chaired major IEEE conferences, such as WCNC 2006, WCNC 2009, VTC 2011 , ICC 2013, and EUSIPCO 2016, and presented tutorial/overview lectures at international conferences. He has presented a number of named lectures and keynotes.

Dr. Hanzo is a fellow of the IEE/IET, the Royal Academy of Engineering, and the EURASIP, a Wolfson Fellow of the Royal Society, and a foreign member of the Hungarian Academy of Science. From 2009 to 2013, he was the Editor-in-Chief of the IEEE Press. He is an IEEE Distinguished Lecturer of the Vehicular Society. He is also the Governor of the IEEE VTS and ComSoc. He has received a number of distinctions, most recently the IEEE Wireless Technical Committee Achievement Award in 2007, the IET Sir Mont Finniston Achievement Award across all disciplines of engineering in 2008, and an Honorary Doctorate of the Technical University of Budapest in 2010 and the University of Edinburgh in 2015. His most recent paper awards include the WCNC 2007 Hong Kong, the ICC 2009 Dresden, the ICC 2010 Cape Town, the WCNC 2013 Shanghai, and the VTC 2014 Vancouver. 


\section{AUTHOR QUERIES}

\section{AUTHOR PLEASE ANSWER ALL QUERIES}

PLEASE NOTE: We cannot accept new source files as corrections for your paper. If possible, please annotate the PDF proof we have sent you with your corrections and upload it via the Author Gateway. Alternatively, you may send us your corrections in list format. You may also upload revised graphics via the Author Gateway.

AQ:1 = Please provide the university for the "master's and D.Sc. degrees." 